\title{
Protocol
}

\section{Presentations of Complex Mental IIIness in Media and Medical Discourses: A Protocol for a Corpus-Assisted Study}

\author{
Laura A. Cariola ${ }^{1}$ \\ University of Edinburgh
}

\begin{abstract}
This study aims to conduct an in-depth corpus-assisted discourse analysis to explore how complex mental illnesses are presented in UK newspaper articles and medical case studies. Attention will be given to the identification of discursive presentation that create and reinforce stigma against mental illness. This study is funded by the Institute for Advanced Studies in the Humanities, University of Edinburgh.
\end{abstract}

\section{Rational and background to the study}

This study aims to conduct an in-depth corpus-assisted discourse analysis to explore how complex mental illnesses, including schizophrenia and bipolar disorder, are presented in UK newspaper articles and medical case studies. Special attention is given to identify how discourse types compare in their communication of stereotypes and prejudices that create and reinforce existing social stigma against individuals affected by mental illness.

Individuals with mental illness are one of the most vulnerable groups in society. For example, one in four people in the UK will experience a mental health problem in any given year (Mental Health Foundation, 2015). It is also a well-known fact that a vast majority of people with mental health issues are exposed to stigma and discrimination, which substantially impacts on their quality of life and recovery (Reavley, Morgan \& Jorm, 2016). Although the Equality Act 2010 makes it illegal to discriminate directly or indirectly against people with mental health issues, the media often links complex mental illness with violence, or portrays people with mental health issues as dangerous, or very disabled and unable to live normal, fulfilled lives (Mental Health Foundation, 2015) - for example, in a large public survey, when asked how to describe someone who has a mental illness, nearly $40 \%$ agreed that they are prone to violence, when in reality people with mental illness are far more likely to be the victims of crime rather than the perpetrator (TNS BMRB, 2015). As such, public discourses are complicit in perpetuating stigma, which impacts on help-seeking

${ }^{1}$ Correspondence concerning this article should be addressed to Dr. Laura A. Cariola, School of Health in the Social Sciences, University of Edinburgh, Teviot Place, Edinburgh EH8 9AG. E-mail: laura.cariola@ed.ac.uk

Language and Psychoanalysis, 2017, 6 (2). Online First published online

$14^{\text {th }}$ June 2017 http://dx.doi.org/10.7565/landp.v6i2.1575 
behaviour and marginalises people with mental health issues as an "undesirable" outgroup within our society (e.g., Clement et al., 2015; Mestdagh \& Hansen, 2013).

Such stigma has been also identified in a pilot corpus-assisted study that explored discourses of borderline personality disorder in UK newspaper articles (Cariola, 2017). Although early parental losses and trauma were reported in both women and men, the results identified highly gendered discourses with significantly more references to women who were portrayed as suicidal and offenders of matricide, and whose identities were constructed through unresolved familial conflicts and dependencies. This alludes to parental conflicts as the cause of developing borderline personality disorder (Whalen et al., 2014). Women were also characterised in relation simplistic life-death existential themes and as passive victims of others' coercive or destructive behaviour. A sample of medical case studies of borderline personality disorders also used gendered discourses where female patients were discussed twice as often than male patients. Such stereotypical gender-based perceptions of borderline personality disorder reinforce the public's negative attitudes towards mental illness and may also interact with clinical perceptions (Benson, Donnellan \& Morey, 2017).

\section{Societal Importance}

Around 13 million people will have a common diagnosable mental disorder in any given year (GOV, 2017) and stigma towards mental health is affecting a large proportion of society. Although public awareness of mental illness has grown and stigma has been reduced, it continues to exist, preventing vulnerable people in need of mental health care from receiving the help they need. Out of this context, the study is timely in addressing a current issue of great societal impact. The exploration of stigma of mental illness, and how it is affecting and shaping the lives of those affected by it, is of great relevance to the field of social science, including clinical psychology (Thoits, 2016).

\section{Methodology and Analysis}

This study is novel in using in-depth corpus-based discourse analysis to examine how UK newspaper articles and medical texts represent discourses of complex mental illness, including schizophrenia and bipolar disorder. It does this by quantitatively identifying semantic content and linguistic patterns in mental health discourses with the use of semantic annotation software packages, such as Wmatrix (Rayson, 2008). Such a semantic analysis provides insight into how word combinations are used to create and reinforce positive and negative connotations, as well as attitudinal and evaluative meanings that impact on public beliefs and stereotypes towards mental illness. Given that violent incidents (such as suicide and homicide) associated with mental health become media headlines, this study will also identify newsworthiness (Bednarek \& Caple, 2017) and its implications to ordinary mental health suffering and recovery. Press articles will be sourced using the web-based library platform LexisNexis that hosts an archive of national newspapers. Medical case studies will be sourced from medical online databases. To provide a representative sample of media publications, this study will focus on UK Broadsheet and Tabloid newspapers of the last 20 years (1997-2017) to explore discursive presentations of mental health and 
diachronic changes of these discourses. These will be contrasted with discourses of published medical case studies that explore autobiographical case histories of patients.

\section{Ethics and Dissemination}

This study has been ethically approved by the University of Edinburgh. The results of this study will be disseminated through publication in peer-reviewed journals as well as via professional conferences and public engagement events. The results of this study will be also discussed in relation to mental health policies and guidelines, and also implications for future research.

\section{Additional Information}

\section{Funding}

This study was funded by the Institute for Advanced Studies in the Humanities, University of Edinburgh, Hope Park Square, Edinburgh, EH8 9NW, Scotland.

\section{Conflict of Interests}

The author has no conflict of interest.

\section{Data Sharing Statement}

This is a study protocol.

\section{References}

Bednarek, M. \& Caple, H. (2017). The discourse of news values: How news organizations create newsworthiness. Oxford, UK: Oxford University Press.

Benson, K. T., Donnellan, M. B., \& Morey, L. C. (2017). Gender-related differential item functioning in the DSM-IV/DSM-5-III (alternative model) diagnostic criteria for borderline personality disorder. Personality Disorder, 8, 87-93.

Cariola, L. A. (2017). Presentations of borderline personality disorder in the UK press: A corpus-assisted discourse analysis. Institute of Group Analysis, London, May 2017.

Clement, S., Schauman, O., Graham, T., Maggioni, F. et al. (2015). What is the impact of mental health-related stigma on help-seeking? A systemic review of quantitative and qualitative studies. Psychological Medicine, 45, 11-27.

GOV (2017). The Government's response to the five-year forward view of mental health. Retrieved from

https://www.gov.uk/government/uploads/system/uploads/attachment_data/file/5 82120/FYFV_mental_health_government_response.pdf

Mental Health Foundation (2015). Fundamental facts about mental health. London, UK: Mental Health Foundation. Retrieved from https://www.mentalhealth.org.uk/sites/default/files/fundamental-facts-15.pdf 
Mestdagh, A., \& Hansen, B. (2014). Stigma in patients with schizophrenia receiving community mental health care: a review of qualitative studies. Social Psychiatry and Psychiatric Epidemiology, 49, 79-87.

Rayson, P. (2008). From key words to key semantic domains. International Journal of Corpus Linguistics, 13, 519-549.

Reavley, N. J., Morgan, A. J., \& Jorm, A. F. (2016). Predictors of experiences of discrimination and positive treatment in people with mental health problems: findings from an Australian national survey. Social Psychiatry and Psychiatric Epidemiology, 52, 269-277.

Thoits, P. A. (2016). "I am not mentally ill". Identifying deflection as a form of stigma resistance. Journal of Health and Social Behaviour, 57, 135-151.

TNS BMRB (2015). Attitudes to mental illness research report 2014. London, UK: TNS-BMRB. Retrieved from:

https://www.time-to-

change.org.uk/sites/default/files/Attitudes_to_mental_illness_2014_report_final 0.pdf

Whalen, D. J., Scott, L. N., Jakubowski, K. P., McMakin, D. L., Hipwell, A. E. et al., (2014). Affective behavior during mother-daughter conflict and borderline personality disorder severity across adolescence. Personality Disorders: Theory, Research, and Treatment, 5, 88-96. 